FORMATION Formation emploi

Revue française de sciences sociales

131 | Juillet-Septembre 2015

Le Bac Pro a 30 ans

\title{
Postface : Le baccalauréat professionnel trente ans après sa création : de la marche forcée à la banalisation?
}

Fabienne Maillard

\section{CpenEdition}

Journals

Édition électronique

URL : http://journals.openedition.org/formationemploi/4518

DOI : 10.4000/formationemploi.4518

ISSN : 2107-0946

Éditeur

La Documentation française

Édition imprimée

Date de publication : 15 octobre 2015

Pagination : 169-187

ISSN : 0759-6340

Référence électronique

Fabienne Maillard, «Postface : Le baccalauréat professionnel trente ans après sa création : de la marche forcée à la banalisation? », Formation emploi [En ligne], 131 | Juillet-Septembre 2015, mis en ligne le 15 octobre 2017, consulté le 10 décembre 2020. URL : http://journals.openedition.org/ formationemploi/4518; DOI : https://doi.org/10.4000/formationemploi.4518

(C) Tous droits réservés 


\title{
Postface
}

\section{Le baccalauréat professionnel trente ans après sa création : de la marche forcée à la banalisation ?}

\author{
FABIENNE MAILLARD \\ Sociologue, professeur des universités, université Lille 3, \\ Proféor-CIREL (Centre interuniversitaire de recherche sur l'éducation)
}

Encore jeune dans l'offre de diplômes du ministère de l'Éducation nationale, puisqu'il a seulement trente ans, le bac pro a échappé, jusqu'en 2009, au mouvement constant de réorganisation qui affecte les diplômes professionnels de ce ministère, depuis leur origine (Brucy, 1998 ; Maillard, 2007). Son évolution apparaît en effet plutôt linéaire malgré toutes les critiques apportées à la politique éducative dans laquelle il a pris forme (conduire $80 \%$ d'une classe d'âge au niveau du baccalauréat en 2000), et à ses propres raisons d'être. Élément d'une réforme qui « a remodelé la morphologie du système scolaire" (Prost, 2011, p. 114), le bac pro a d'emblée été présenté comme une innovation par ceux qui l'ont institué... et comme un diplôme ambigu par la plupart des chercheurs qui s'y sont intéressés. Cette appréciation tient à des caractéristiques spécifiques qui le distinguent aussi bien des autres baccalauréats que des diplômes professionnels avec lesquels il cohabite : le CAP (Certificat d'aptitude professionnelle), le BEP (Brevet d'études professionnelles) et le BP (Brevet professionnel).

Sa première particularité, et non des moindres, est d'être un baccalauréat dédié à l'insertion professionnelle. Si cette finalité a immédiatement été jugée difficilement compatible avec la définition du baccalauréat comme premier grade de l'enseignement supérieur, elle avait néanmoins pour objectif de désenclaver l'enseignement professionnel, alors réduit au "second cycle court ${ }^{1}$, et de lui redonner les lettres de noblesse que son intégration dans le système scolaire, dans les années 1960, lui avait fait perdre. Deux autres traits en font un diplôme original : l'obligation, pour ceux qui le préparent, d'immersion dans les entreprises via des périodes de formation en milieu professionnel (PFMP) de plusieurs semaines, qui instituent "l'alternance sous statut scolaire ", et l'évaluation par contrôle en cours de formation (CCF).

C'est justement ce caractère innovant qu'interroge le dossier de Formation Emploi consacré au bac pro (1995), dix ans après sa naissance. À l'époque, la définition du diplôme, sa singularité et ses objectifs posent encore question. Le bac pro, qui s'est

1. Le CAP et le BEP relevant du niveau V et le BP n'étant pas accessible aux élèves mais seulement aux adultes et, depuis 1992, aux apprentis. 
considérablement développé dans la voie professionnelle grâce à une politique très volontariste, apparaît toujours incongru dans le paysage des diplômes.

Vingt ans après cette publication emblématique, la seule jusquà présent consacrée entièrement à ce diplôme, le bac pro a changé de position au sein des baccalauréats, son cursus a été transformé et la poursuite d'études est devenue l'une de ses missions clairement affichée. Officiellement, il a été mis à parité avec les autres baccalauréats.

Trois bacheliers sur dix sont désormais titulaires d'un bac pro, contre deux sur dix d'un baccalauréat technologique. Le bac pro est le deuxième baccalauréat par le volume de ses titulaires. Ce diplôme se prépare en outre en trois ans directement après la troisième, comme les baccalauréats général et technologique, et non plus en quatre ans en passant par un BEP.

Bien que le ministère désigne la réforme à l'origine de ces changements, engagée en 2007 et généralisée en 2009, comme une simple " rénovation " de la voie professionnelle, c'est bien une métamorphose qui a eu lieu.

À l'occasion du trentenaire du diplôme, ce nouveau numéro de Formation Emploi permet de revenir sur les évolutions et les perceptions du bac pro. Est-il désormais devenu un diplôme comme les autres, dont l'originalité et les fonctions se sont banalisées ?

Pour tenter de répondre à cette question, la réflexion esquissée ici confronte différents travaux et propose quatre parties. Les spécificités du bac pro, que les chercheurs ont surtout envisagées comme des paradoxes, sont au cour de la première. La légitimité du diplôme en tant que baccalauréat s'est ainsi révélée être une question récurrente, comme l'a été sa dimension ouvrière, qu'interroge la deuxième partie. Objet d'un intérêt soutenu, le traitement du public scolaire, abordé dans la troisième partie, a davantage scindé les approches, les unes insistant sur la relégation scolaire des lycéens professionnels, les autres sur leur réhabilitation. Depuis la reconfiguration du diplôme, un nouveau champ des possibles semble toutefois offert aux bacheliers professionnels, dont rendent compte les articles présentés dans ce numéro. Un certain rapprochement avec les autres baccalauréats serait ainsi en train de prendre forme, perspective que discute la dernière partie.

\section{Un diplôme désigné par ses ambiguïtés}

Il a fallu quelques années pour que les chercheurs s'intéressent au bac pro. Parmi les auteurs des premiers articles sur le sujet figure ainsi fréquemment Benoît Bouyx, qui fut l'un des artisans de la création du bac pro en tant que Secrétaire général des Commissions professionnelles consultatives du ministère de l'Éducation nationale. Comme ces commissions paritaires ont la responsabilité de définir les spécialités de 
chaque diplôme et leurs référentiels, il en était un observateur privilégié. Pour lui, cette création est "le résultat d'une heureuse rencontre entre la demande du monde économique et la volonté de l'Éducation nationale de décloisonner (certains diront sortir du ghetto) les formations professionnelles de type CAP-BEP» (1996, p. 75).

Une telle interprétation enchante une décision qui fut à la fois impérative et contestée. Plus prosaïquement, cette décision est issue de la volonté de trouver les moyens de répondre aux ambitions de la politique éducative, orientées vers une hausse considérable du niveau général d'éducation, et de retarder l'entrée dans la vie active des plus jeunes, à un moment où le chômage juvénile croissait dangereusement et atteignait les diplômés du niveau V (Prost, 2011).

S'agissant de la réponse aux demandes économiques, les avis sont partagés. La demande formulée par l'Union des Industries Métallurgiques et Minières (UIMM), qui a permis de justifier l'initiative de création du nouveau baccalauréat, ne visait pas la création d'un nouveau baccalauréat et aurait pu trouver une autre réponse.

Quant aux secteurs d'activité dans lesquels le bac pro a aussitôt pris place, ils n'étaient pas non plus aussi unanimes que ce qu'en a laissé penser le ministère, si l'on en croit Michel Pillet, qui s'est penché sur la genèse des cinq premières spécialités du diplôme (1995). Cet auteur montre également que derrière la perspective d'élever la qualification de la main-d'œuvre pouvaient se cacher d'autres stratégies, comme la volonté de résoudre un déficit d'image, ce qui était le cas pour la "vente-représentation ». En décryptant la genèse des trois premiers bacs pros de la métallurgie, Bernard Fourcade (1997) met pour sa part en valeur les différents compromis dont ils résultent : entre les impératifs institutionnels et les revendications des employeurs, entre les diverses interprétations proposées des mutations techniques et organisationnelles, ainsi qu'entre les fédérations patronales et les branches impliquées. Le bac pro «Équipements et installations électriques" (devenu plus tard Électrotechnique), qui relève en même temps de la métallurgie, du bâtiment et des services, a par exemple profondément divisé les différents acteurs de la négociation.

Les organisations professionnelles n'étaient donc pas toutes aussi favorables à la création du bac pro que ce qu'ont pu en dire les responsables politiques et institutionnels.

Il existait également des dissensions au sein du gouvernement sur la politique des $80 \%$ et sur l'opportunité de créer un baccalauréat dans la voie professionnelle. Lucie Tanguy rappelle que c'est en raison d'un désaccord avec la politique " de niveau ", axée sur l'élévation du niveau de formation en France, que le Secrétariat d'État à l'enseignement technique lui a commandé, en 1990, un rapport sur les formations à développer pour les ouvriers et les employés ${ }^{2}$ (2013). De fait, « la légitimité des formations professionnelles

2. Publié en 1991 à La Documentation française. 
conduisant à des emplois d'ouvriers et d'employés s'est trouvée mise en cause " par cette politique éducative (ibid., p. 43).

La création du bac pro a néanmoins été parée d'une rhétorique adéquationniste, qui pouvait d'autant plus être entendue que la perspective d'une "moyennisation" de la société était au goût du jour (Chauvel, 1998), et que les transformations engagées dans les process de production et les organisations du travail semblaient aller dans le sens d'une importante hausse du niveau des qualifications professionnelles.

Résultat d'une procédure de décision extrêmement rapide désavouée par les organisations patronales et les syndicats enseignants parce qu'elle s'est passée de dialogue social, le diplôme prend très vite son essor. Cette progression, analysée par les responsables politiques comme la conséquence d'une forte "demande sociale ", fait de l'instauration du bac pro un succès, qu'aucun des ministres qui se succéderont à la tête de l'Éducation nationale ne remettra en cause.

Une telle célérité n'est cependant pas seulement due aux espoirs suscités par ce nouveau diplôme mais aussi aux efforts engagés par le ministère. En plus de la création successive de nouvelles spécialités, des consignes sont envoyées aux académies pour favoriser l'ouverture de sections de bac pro, au prix de la fermeture de sections de CAP. Engagée à moyens constants, la politique de mise en place du bac pro repose en effet sur l'élimination du CAP dans les lycées professionnels et sur l'expansion du BEP, diplôme qu'il faut alors posséder pour entrer en bac pro. Ces mesures imposent d'importants mouvements dans la voie professionnelle, au point de la reconfigurer totalement.

Elles seront suivies par d'autres mesures tout aussi volontaristes, qui modifieront à nouveau la structure et les curricula des diplômes, mais en suivant des directions contradictoires. Après avoir subi un mouvement d'éradication, le CAP est en effet refondé en 2002, tandis que le BEP devient un diplôme "propédeutique ", destiné à conduire au bac pro (Maillard, 2005). En 2009, au moment où intervient la réforme de la voie professionnelle, le CAP est une nouvelle fois relancé car c'est désormais le seul diplôme qui forme au niveau $\mathrm{V}$, le cursus de formation au BEP ayant été supprimé.

Après avoir servi de rampe de lancement au bac pro, le BEP est accusé de ne pas y conduire assez d'élèves et de constituer un obstacle à la croissance du bac pro, comme à l'atteinte de l'objectif des $80 \%$ au niveau du bac. Il ne disparaît pas pour autant de l'offre de diplômes : doté d'un curriculum et d'épreuves "allégés» (le terme est officiel), il subsiste en tant que "diplôme intermédiaire" (Maillard, 2011).

En 1990, les élèves qui préparent un bac pro sont déjà près de $94000^{3}$, auxquels s'ajoutent plus de 4000 apprentis. Car l'apprentissage s'intéresse lui aussi à ce diplôme, à un moment où le CAP n'a pas bonne presse et où de nouvelles opportunités s'offrent

3. Ce chiffre comme les suivants proviennent de diverses éditions de Repères et références statistiques du ministère de l'Éducation nationale. 
aux centres de formation d'apprentis, grâce à la loi de $1987^{4}$. Le nombre de spécialités ne cesse pas non plus de croître. De cinq en 1985, elles sont passées à une vingtaine en 1990 et sont, en 2015, près d'une centaine.

Impulsé par le ministère, ce développement a progressivement été sollicité par les branches professionnelles, celles qui s'étaient montrées les plus réticentes à l'égard du diplôme ayant fini par se convertir à la nouvelle offre de formation (comme la coiffure très récemment). Il faut dire que l'absence de bac pro dans un domaine de spécialité apparaît aujourd'hui plutôt pénalisante et fait planer un soupçon d'archaïsme sur le domaine en question.

Bien qu'il ait été épargné par l'entreprise réformatrice du ministère jusqu'en 2007, le bac pro a été un important déclencheur d'évènements. Il a été et reste au cœur de bouleversements profonds dans la voie professionnelle et donc dans le système éducatif. Il a également suivi quelques évolutions notables, puisqu'il était prévu au départ de privilégier la dimension transversale du diplôme et de ne pas dépasser une cinquantaine de spécialités. Or, le bac pro en comprend désormais beaucoup plus et peut être un diplôme très spécialisé. Différentes logiques ont donc présidé à la création de nouvelles spécialités. Il serait par conséquent intéressant d'en savoir plus, afin d'aller au-delà des contradictions repérées par les chercheurs à propos de la genèse du diplôme et de ses premières spécialités.

Si la connaissance du bac pro est encore parcellaire, elle a également mis quelque temps avant de s'accumuler. Elle a d'abord reposé sur les publications statistiques du ministère et du Céreq (Centre d'études et de recherches sur les qualifications), avant de mobiliser les chercheurs. Ainsi, la thèse de doctorat de Georges Solaux, consacrée à ce diplôme (1990), n’a pas été publiée. C'est seulement au début des années 1990 que le bac pro devient un objet partagé de la réflexion scientifique. Il ne s'agit pas forcément, cependant, d'appréhender un diplôme pluriel dont l'expansion pose de nouvelles questions, mais plutôt de souligner les ambivalences de la politique éducative, qui sont aussi celles du diplôme. Sa cible professionnelle, technicien ou ouvrier très qualifié, est ainsi au centre de plusieurs travaux.

Philippe Zarifian (1993) souligne, par exemple, les contradictions des représentants de l'État sur ce point, en estimant que les emplois d'ouvriers et d'employés auxquels ont et auront accès les bacheliers professionnels sont les mêmes que ceux des titulaires de $\mathrm{CAP}$ et de BEP, dont ils prendront la place. Il évoque dès lors la situation de " déclassement " en chaîne des diplômés, déclassement qui sera bientôt au cœur des analyses portant sur ce diplôme. Dans le même numéro de l'Orientation scolaire et professionnelle, Henri Eckert et Bernard Hillau mettent en cause la diversification du niveau

4. Cette loi du 23 juillet 1987 sur l'apprentissage ouvre aux CFA (centres de formation d'apprentis) la possibilité de préparer tous les diplômes professionnels, du niveau $\mathrm{V}$ au niveau I. 
IV et l'introduction "dans les professions d'ouvriers et d'employés, de nouvelles références scolaires" (1993, p. 166).

Malgré les innovations dont le bac pro est porteur sur les plans institutionnel et pédagogique, ce que montre Georges Solaux en analysant son curriculum (1995), ses dissonances focalisent davantage l'attention des chercheurs. À l'instar de Catherine Agulhon, auteur de travaux pionniers sur le bac pro (1994, 1995 \& 1997), Myriam CampinosDubernet dresse ainsi "un constat mitigé " sur les évolutions du diplôme (1995, p. 3). S'il ouvre l'accès au baccalauréat à de nouvelles fractions de jeunes des milieux populaires, il contribue en même temps à sédimenter la hiérarchie des savoirs, se révèle plus technique que professionnel, et ne s'est pas imposé sur le marché du travail. Mais alors que l'association entre bachelier et ouvrier est la plupart du temps décriée, Myriam Campinos-Dubernet estime que « la création de l'ouvrier bachelier est peut-être effectivement une chance de maintenir à cette catégorie sociale (celle des ouvriers) une légitime dignité» (1995, p. 27).

Le constat unanime des ambiguïtés du diplôme conduit à des interprétations divergentes. Toutefois, c'est surtout vers la dénonciation d'un faux baccalauréat et d'un faux diplôme de technicien que tendent les approches.

Madeleine Figeat assimile ainsi le bac pro à " un leurre ", en estimant qu'il produit de faux espoirs, puisqu'il ne mène ni aux emplois annoncés ni à la poursuite d'études (1996). Cette caractérisation négative, qui associe volontiers le bac pro à un leurre ou une illusion, demeure vivace trente ans après. Elle n'est cependant pas propre au bac pro et n'a épargné aucun diplôme professionnel destiné aux emplois d'exécution, même les plus qualifiés (Brucy, 1998).

Toujours suspects de faiblesse scolaire, de subordination au patronat, d'excès de scolarisation ou au contraire de spécialisation professionnelle, et donc de décalage avec l'emploi (les reproches varient selon les diplômes, selon ceux qui les formulent mais également dans le temps), les diplômes associés à l'enseignement professionnel ${ }^{5}$ apparaissent trop orthogonaux à l'égard de la norme scolaire pour que leur légitimité soit garantie. L'une des conséquences de leur double définition et de leurs multiples fonctions est qu'ils ne sont jamais « ce qu'ils devraient être".

5. Dont on oublie la plupart du temps, en raison d'un tropisme très scolarocentré, qu'ils rassemblent plusieurs milliers d'apprentis et d'adultes qui les préparent par la formation continue, une candidature libre, le Centre national d'enseignement à distance ou la validation des acquis de l'expérience. 


\section{Un diplôme ouvrier?}

Une bonne part des analyses adressées à la politique éducative et au diplôme s'en prennent aux fausses promesses des responsables politiques. La cible professionnelle du bac pro était en effet censée se distinguer de celle des diplômes de niveau V, ce qui s'est avéré pour les premières promotions de sortants, mais pas pour les suivantes.

Cette posture critique repose largement sur une conception industrielle et ouvrière $\mathrm{du}$ bac pro, encore très active dans les travaux des chercheurs. Elle était déjà présente dans L'école capitaliste en France (Baudelot et Establet, 1971) et dans L'ordre des choses, ouvrage dans lequel Claude Grignon (1971) voit dans les futurs diplômés de la voie professionnelle les "petits chefs" asservis de l'industrie. La désouvriarisation de l'enseignement professionnel et l'émergence de l'ouvrier-bachelier mobilisent plusieurs approches, qui s'interrogent sur la construction d'une nouvelle figure ouvrière au service des objectifs managériaux (Beaud, 1996 ; Eckert, 1999).

Dans le présent numéro, Séverine Misset réactualise ces interrogations, pour montrer cependant que les bacheliers professionnels devenus ouvriers peuvent avoir une appropriation positive de la condition ouvrière, contrairement à ce qui en est dit le plus souvent, et qu'ils ne sont pas les plus prompts à imposer les règles managériales, bien au contraire. Ce sont plutôt les ouvriers promus sur le tas qui se révèlent les plus empressés à faire respecter les normes et les objectifs de l'entreprise. Autrement dit, la conversion des bacheliers professionnels ouvriers à la doxa managériale n’est pas accomplie.

Bien qu'il permette de redonner de la substance à une population ouvrière trop souvent négligée par les chercheurs comme par les responsables politiques, ce tropisme industriel des recherches aboutit à dresser un portrait très incomplet de la voie professionnelle et du bac pro. L'enseignement professionnel a pourtant toujours proposé des spécialités tertiaires, lesquelles accueillent, depuis trois décennies, les effectifs d'élèves les plus élevés ${ }^{6}$. Bien que le nombre des spécialités industrielles soit toujours supérieur à celui des spécialités tertiaires, c'est dans ces dernières que l'on enregistre les plus gros flux. À la rentrée 2013, 58.2 \% des élèves inscrits en bac pro préparaient une spécialité des services, contre $41.8 \%$ dans l'industrie. Ces chiffres sont les mêmes qu'en 1998. Du reste, c'est aussi à la professionnalisation des activités d'employé qu'était destinée l'expansion du diplôme, même si, en l'associant lors de sa conception à une qualification de technicien ou d'ouvrier-technicien, le ministère a contribué à forger une vision réductrice du bac pro.

6. En 1986, le nombre des élèves inscrits en CAP et en BEP dans des spécialités tertiaires dépasse celui des élèves des spécialités industrielles, grâce à l'enseignement privé. 
Dans le dossier de Formation Emploi de 1995, consacré aux dix ans du bac pro, Henri Eckert (1995) montre que, parmi les raisons qui expliquent les difficultés d'accès à l'emploi des titulaires du diplôme, après une période favorable, figurent la dégradation de la conjoncture économique mais aussi l'explosion des effectifs dans les formations tertiaires. Or, le secteur tertiaire a particulièrement recours aux emplois aidés et impose volontiers des emplois à temps partiel. La qualification n'y est pas non plus toujours bien circonscrite, défaut que des chercheurs ont tenté de résoudre au début des années 2000, en établissant des critères pour distinguer les employés qualifiés des non-qualifiés, ce que l'INSEE ne faisait pas'.

Alors que pendant les années 1990, les critiques des chercheurs se polarisent sur les écarts entre les discours officiels et les emplois occupés par les bacheliers industriels, c'est néanmoins dans les spécialités industrielles que les conditions d'insertion sont les plus favorables. En 2015, ce résultat est toujours valide, bien que le recours aux emplois aidés se soit banalisé et s'applique largement aux sortants du système éducatif, même lorsqu'ils possèdent un diplôme.

En raison de cette indétermination de la qualification visée par le bac pro (indétermination qui concerne plus généralement le niveau IV de formation), son " espace de qualification " pouvait être considéré comme "flou " dix ans après la création du diplôme (Campinos-Dubernet, 1995). Le diagnostic était sévère, mais les enquêtes les plus récentes conduites par le Céreq n'invitent pas à en changer. La croyance dans la hausse inéluctable des qualifications professionnelles et dans la fin des emplois d'exécution a fait long feu. Plus de la moitié de la population active occupe encore aujourd'hui un emploi d'ouvrier ou d'employé, la structure des niveaux de formation des sortants du système éducatif ayant changé bien plus rapidement que la structure des qualifications dans le système d'emploi.

L'argument de la nouveauté est souvent brandi pour réclamer un diplôme, une spécialité ou un changement de référentiel. Il est fréquemment utilisé par les partenaires sociaux qui participent à la définition des diplômes et des référentiels au sein des commissions professionnelles consultatives. Le ministère s'en empare quand ça l'arrange, quitte à ne pas entendre d'autres arguments. Dans les formations industrielles, les inspecteurs se montrent particulièrement sensibles à l'attraction du changement, qui peut les conduire au déterminisme technologique (Veneau, 1997 ; Quenson, 2009). Philippe Mouy et Patrick Veneau ont ainsi rapidement souligné que la qualification de technicien d'atelier n'était pas accessible aux débutants et qu'elle ne concernait en outre qu'une petite fraction de salariés (1995). Entre l'image que projette le référentiel et la réalité du travail, la distance apparaît par conséquent plutôt grande.

7. Voir les travaux de Chenu, Burnod, Chardon et d'autres. 
Dans la mesure où ce sont les diplômes industriels qui ont surtout intrigué les chercheurs, et où le devenir des bacheliers professionnels n'a été abordé que sur de brèves périodes, de nombreuses questions restent cependant ouvertes.

On peut par ailleurs regretter qu'aucun article du présent dossier n'ait accordé d'attention aux correspondances entre les bacs pros et l'emploi. Alors que la hausse des exigences professionnelles reste un leitmotiv dans certaines activités, l'observation de ces dernières invite à une certaine prudence d'interprétation (voir par exemple Cadet et alii, 2014, pour le commerce et la vente).

Au moment où le bac pro a été institué, la fin du taylorisme et des emplois d'exécution était annoncée, justifiant non seulement le développement du diplôme mais également l'extinction du CAP.

Trente ans plus tard, il s'avère que le taylorisme s'est répandu là où on ne l'attendait pas, tout en se maintenant avec force dans certaines activités (industries agro-alimentaires et restauration rapide par exemple). Quant à l'emploi non qualifié, qui devait rapidement diminuer, il a connu une forte recrudescence dans les années 1990 et rassemble plus de cinq millions d'actifs occupés, selon l'INSEE.

\section{Des recherches également 3 plutôt centrées sur les élèves}

Si l'attention des chercheurs pour la genèse des diplômes et leur curriculum a marqué le pas, ce n'est pas le cas s'agissant des jeunes en formation. Ces derniers ont toujours beaucoup intéressé les chercheurs qui se sont penchés sur la voie professionnelle, et qui se divisent sur l'interprétation à donner de son rôle de remédiation scolaire et de formation (Agulhon, 1994 ; Moreau, 1995 ; Beaud, 1996 ; Jellab, 2001 \& 2008 ; Palheta, 2011 ; Bernard et Troger, 2012 ; Dagot et Dassié, 2014).

Alors que la voie professionnelle représentait un espace de promotion scolaire et sociale jusqu'à la fin des années 1950, les nouvelles attributions qui lui ont été confiées pour généraliser l'accès au second cycle du second degré lui ont fait perdre son statut, son autonomie, ainsi que son public, auparavant soigneusement trié sur le volet.

Depuis lors, c'est comme instance de "relégation " qu'elle est perçue, conception que les politiques publiques ont construite tout en arguant régulièrement de leur volonté de " revaloriser " cette voie de formation ; relégation liée à son public d'exclus des autres filières, à ses diplômes particuliers et aux emplois auxquels ils destinent : certes a priori qualifiés mais confinés dans les catégories d'exécution. Dans la hiérarchie scolaire, la voie professionnelle est située en bas. 
Malgré la création du bac pro, la double relégation des élèves, dans le système éducatif et dans l'emploi, reste un thème cher à bien des auteurs, qui l'envisagent en termes de "domination" (Palheta, 2012 ; Depoilly, 2014). Le fait que les diplômes professionnels ne mènent "qu’à " des emplois déclarés "subalternes " (bien qu'ils soient pour le moins aussi bien différents qu'inégaux) inscrit ces travaux dans une tradition critique de la sociologie de l'éducation, inspirée par les travaux de Pierre Bourdieu et JeanClaude Passeron sur La reproduction (1970). Si elle n'est pas inédite, cette approche invite néanmoins à remettre en question l'ordre scolaire et le rôle du système éducatif dans la division du travail. Elle réactualise un débat qui était très vif dans les années 1970, en y incorporant les critères de genre, plutôt délaissés par les sociologues de l'époque. Cependant, tout en confirmant des résultats antérieurs, les approches par la relégation tendent souvent à sous-estimer la force des rapports sociaux et la dimension historique du social. Une certaine inertie de l'enseignement professionnel y transparait. Il arrive aussi, et c'était un reproche adressé à Pierre Bourdieu et à Jean-Claude Passeron, qu'elles survalorisent l'influence de l'école sur le système d'emploi et méconnaissent le fonctionnement du marché du travail. La différenciation des diplômes, de leurs publics et les effets produits par les innombrables rénovations imposées par les pouvoirs publics n'y sont pas non plus considérés, ce qui tronque l'image donnée de la voie professionnelle, même réduite au seul enseignement professionnel.

Lorsqu'ils s'intéressent aux élèves des lycées professionnels, les chercheurs ne les distinguent pas forcément selon les diplômes et les spécialités préparés. Catherine Agulhon a pourtant mis en valeur leurs multiples disparités (1994), qui sont loin d'être seulement superficielles. Si "la jeunesse n'est qu'un mot ", comme l'affirme très justement Pierre Bourdieu, c'est également vrai pour la jeunesse populaire inscrite dans la voie professionnelle. La condition sociale et la condition scolaire que partagent les jeunes par leur appartenance massive aux catégories ouvrières et employées et leur inscription dans une filière qui accueille les jeunes en difficultés scolaires ne sont en effet ni homogènes ni indivisibles. De même que les "dominés " ne sont jamais seulement dominés, ils ne composent pas une population uniforme et unifiée, même si l'échec scolaire, une orientation par défaut et un certain rapport au savoir peuvent caractériser les jeunes accueillis dans la voie professionnelle (Charlot, 1999).

D'emblée, la voie professionnelle se divise entre l'enseignement professionnel et l'apprentissage, ce qui n'est pas une mince distinction. L'apprentissage se révèle ainsi plus industriel, masculin et blanc (Moreau, 2003). Quant à l'enseignement agricole, il constitue lui aussi un segment particulier, sans être homogène pour autant. Laure Minassian montre, dans ce numéro, que l'on peut y distinguer deux réseaux de scolarisation : primaire-professionnel et secondaire-supérieur, qui participent activement à la production d'inégalités scolaires et sociales.

Multiforme, la voie professionnelle est également stratifiée, par ses diplômes, dont le niveau de formation et la position institutionnelle diffèrent, comme par ses spécialités. 
Dans l'ensemble des bacs pros, par exemple, certains sont plus attractifs que d'autres et le champ des possibles qu'ils ouvrent se révèle très différent selon les spécialités de formation, que ce soit en termes de poursuite d'études ou d'emploi. Ils ont également des images et des réputations, qu'entretiennent ou combattent leurs concepteurs au moment des rénovations, et dont on retrouve certaines traces dans les référentiels (Maillard, 2013). Tous ne forment pas non plus le même type de public, certains pouvant imposer une certaine sélection (comme dans les métiers d'art), alors que d'autres accueillent les jeunes qui n'ont pas trouvé de place ailleurs. Les formations mixtes ne sont pas non plus très nombreuses et adhèrent bien au genre des emplois qu'elles visent. L'image des formations reste cependant labile et varie selon les régions, les lieux d'implantation des établissements et les établissements, leur inscription dans le tissu local et leur public. La chaudronnerie, qui peine à remplir les sections où elle est enseignée, peut ainsi trouver grâce aux yeux de certains jeunes assurés de trouver un emploi de proximité avec leur bac pro (Cizeau, 2013). De tels écarts, cependant, sont plus souvent relevés par les enquêtes sur les modes d'accès à l'emploi des jeunes, qui cherchent à mesurer l'influence de nombreuses variables, que par des recherches plus qualitatives.

Cet attrait des chercheurs pour les élèves, plus ample que pour les apprentis, est encore une fois démontré avec ce numéro. Alain Thalineau et Florian Hot s'intéressent ainsi aux liens entre le désir de mobilité résidentielle pour accéder à l'emploi et le groupe familial, dont l'influence apparaît déterminante. Axée sur des jeunes en formation, qui ne sont donc pas confrontés immédiatement à une recherche d'emploi, l'enquête réalisée, à la fois quantitative et qualitative, aboutit à mettre en valeur l'effet, sur les perspectives de mobilité, de la cohésion du groupe familial vis-à-vis des attentes de l'école et du monde du travail.

L'influence de la généralisation du bac pro en 3 ans sur la scolarité et les aspirations des élèves est également abordée par deux articles, aux analyses plutôt convergentes. La revalorisation de l'enseignement professionnel que la réforme de la voie professionnelle prétendait mettre en œuvre se traduit-elle dans la pratique ? Spécialiste de l'enseignement professionnel, principalement du point de vue des élèves et des enseignants, Aziz Jellab s'interroge sur le rapport au savoir des bacheliers professionnels confrontés à l'incitation à la poursuite d'études. En s'opposant aux analyses qui limitent l'enseignement professionnel à une fonction de reproduction économique et sociale, il montre que de nombreux lycéens professionnels vivent positivement leur expérience scolaire et ne se contentent pas de rationaliser un destin entériné. Il souligne aussi à quel point la spécialité de formation structure le rapport au savoir. Si ces perceptions positives de la scolarité ne découlent pas directement de la réforme du bac pro, celle-ci les favorise en permettant aux élèves de mieux assumer leur orientation et en ouvrant le champ des possibles. Le bac pro n'est plus envisagé comme une fin mais comme une étape dans un parcours de formation. Toutefois, bien que ces nouvelles perspectives 
offertes aux bacheliers professionnels invitent à considérer qu'une revalorisation de la voie professionnelle a bien eu lieu, Aziz Jellab signale en même temps que la volonté de poursuite d'études pourrait paradoxalement renforcer la disqualification sociale et professionnelle des bacheliers professionnels qui entrent dans la vie active. Dès lors que l'accès à l'enseignement supérieur devient une norme étendue à l'ensemble des voies de formation, chercher un emploi après l'obtention du baccalauréat pourrait représenter un signe d'échec.

La recherche que j'ai réalisée sur les premiers effets de la réforme de la voie professionnelle m'induit à souligner l'importance de ce risque, qui relativise la revalorisation engagée (Maillard, 2010). La dénégation de la finalité professionnelle des diplômes "professionnels » ne peut être seulement envisagée comme une avancée. Elle a en effet largement contribué au déclin du CAP lorsqu'il était désigné comme un diplôme de remédiation scolaire, avant de conduire au discrédit du BEP, puis à l'élimination de son cursus de formation. Devenu propédeutique au début des années 2000, le BEP restait néanmoins appréhendé par les jeunes qui l'obtenaient comme un diplôme professionnel, soit un diplôme ouvrant sur la vie active. Cette finalité, a priori rationnelle tant une définition exclusivement propédeutique d'un diplôme professionnel peut paraître inadaptée, a cependant été jugée inappropriée par le ministère, les sorties postBEP finissant même par être assimilées à des décrochages scolaires. En insistant sur les liens entre le bac pro et le BTS (brevet de technicien supérieur), dans le contexte d'une politique éducative dite "de bac moins 3 à bac plus 3 ", il se peut que la politique éducative fragilise davantage la position du bac pro sur le marché du travail, sans parvenir à garantir sa mise à parité avec les autres baccalauréats. C'est pourtant l'une des promesses de la réforme de la voie professionnelle.

Pour Pierre-Yves Bernard et Vincent Troger, cependant, le pari de revaloriser la voie professionnelle a été tenu. À partir d'une enquête longitudinale conduite auprès de jeunes formés en bac pro, ils trouvent nombre d'avantages au bac pro en trois ans. Outre que cette réforme rend les élèves plus satisfaits de leur orientation, qu'ils ont plus souvent choisie que leurs prédécesseurs, elle les incite à se projeter dans un avenir plus large, où la poursuite d'études apparaît à la fois souhaitable et possible ${ }^{8}$. En passant à trois ans de formation et en assumant clairement sa finalité de poursuite d'études, le bac pro semble s'être rapproché des baccalauréats général et technologique. Dans cette mesure, sa transformation participerait à lever les ambiguïtés jusque-là attachées au diplôme. Est-ce bien sûr ?

8. Ces résultats optimistes gagneraient cependant à être confortés par d'autres analyses, l'enquête que j'ai réalisée au tout début de la réforme (citée plus haut) n'allant pas dans une telle perspective. Tout en ouvrant le débat, ces variations montrent bien les dimensions plurielles de la voie professionnelle et du bac pro. 


\section{Un baccalauréat équivalent aux autres?}

Avec plus de 540000 élèves et près de 63000 apprentis en 2013, le bac pro occupe une place notable dans le système éducatif. Cette position majeure provient d'une politique spécifique, qui a non seulement changé son cursus de formation mais également sa définition.

La poursuite d'études n'est ainsi plus considérée comme une possibilité annexe que le ministère tolère. En 1996, Benoît Bouyx pouvait ainsi affirmer qu' " une fréquence trop importante des poursuites d'études (20\% avaient été envisagés, qui semblent atteints si ce n'est dépassés aujourd'hui) conduirait à dénaturer le baccalauréat professionnel et à lui faire perdre son caractère "professionnel"... C'est ce qui est arrivé à tous les diplômes ayant par le passé affirmé une double finalité "(1996, p. 76). Bien que la mise en garde soit claire et parfaitement justifiée si on la rapporte à l'histoire des diplômes professionnels et techniques, son écho a peu résonné. Depuis 2005 en effet, la fonction propédeutique du bac pro est valorisée, et depuis 2009, elle est même à la source de diverses initiatives pour favoriser la réussite des titulaires du diplôme dans l'enseignement supérieur.

Si elle est aujourd'hui au cour de l'actualité, la question de la poursuite d'études des bacheliers professionnels dans l'enseignement supérieur n'est pas inédite. Elle était cependant traitée plus discrètement par les pouvoirs publics, confrontés à l'ambivalence de leur création. L'accès à l'enseignement supérieur a en effet été très rapidement une tentation pour les titulaires du diplôme, encouragés en cela par leurs enseignants. Dans l'ensemble des diplômes professionnels de l'Éducation nationale, la singularité du bac pro n'était pas à leurs yeux de conduire à l'emploi mais bien aux études supérieures. Cette projection avait cependant le défaut de reproduire l'histoire du baccalauréat de technicien et du brevet de technicien (BT), détournés de leur fonction professionnelle pour ne plus conduire qu'à la poursuite d'études. Or, c'est à la vie active que devait mener le bac pro. Si le décloisonnement de la voie professionnelle était l'un des objectifs de la création du diplôme, il intervenait néanmoins dans un périmètre bien circonscrit.

Bien qu'initialement, comme le rappelle Benoît Bouyx dans son propos, un taux de poursuite d'études de $20 \%$ ait été jugé comme un maximum à ne pas dépasser, il a vite atteint $30 \%$. Dans la mesure où le droit d'accès à l'enseignement supérieur est un attribut du baccalauréat, il était difficile de l'interdire mais pas impossible de le déconseiller fortement. C'est ce qu'a fait le ministère pendant plusieurs années. Faire en sorte que les bacheliers professionnels évitent de poursuivre des études pouvait donc être envisagé, même si c'était plus facile à faire dans les STS (Sections de Techniciens Supérieurs) et les IUT (Instituts Universitaires de Technologie), où la sélection est prévue, qu'à l'université, où elle est encore interdite. Dans cette mesure, le combat mené par le ministère pour réguler les poursuites d'études des bacheliers professionnels ne pouvait qu'être perdu. 
Selon Stéphane Beaud (2002), c'est pour " retrouver la voie normale " que les bacs pros sont allés à l'université, quitte à dénier la faiblesse de leurs résultats scolaires et leur faible investissement dans le travail requis par les études. Ils réclamaient ainsi un droit à être étudiants comme les autres, dès lors que ce statut s'était normalisé. Mais cette orientation s'est révélée "une impasse ", marquant l'échec de ces jeunes comme celui du bac pro en tant que baccalauréat (Beaud \& Pialoux, 2001). Selon plusieurs auteurs, c'est une fois entrés dans l'enseignement supérieur que les bacheliers professionnels prennent conscience des particularités de leur diplôme; l'accusation de " leurre " ne serait donc pas qu'une appréciation de chercheur mais aussi celle des déçus du bac pro, dont le curriculum ne prépare pas aux études supérieures. Tout en se démarquant d'une telle approche, qu'ils contestent même vivement, Aziz Jellab, de même que Pierre-Yves Bernard et Vincent Troger évoquent, dans leurs articles respectifs, le décalage entre les exigences de la formation dans l'enseignement supérieur et les efforts consentis par les jeunes, qui n'ont pas acquis certaines habitudes de travail et souffrent d'un certain manque d'autonomie face au travail intellectuel. Ils peuvent cependant s'y trouver bien, comme l'illustre l'article de Magali Danner et Christine Guégnard, également dans ce numéro, qui sont allées interroger des bacheliers professionnels inscrits dans des classes préparatoires à des écoles supérieures d'art, autrement dit là où l'on ne s'attend pas à rencontrer ces jeunes. Elles renversent elles aussi le fatalisme qui est souvent accolé aux bacs pros devenus étudiants. L'arrivée de ces "nouveaux bacheliers " à l'université y a d'ailleurs très vite été mise en cause, en raison de leur insuffisance scolaire et de leur comportement inapproprié (Blöss \& Ehrlich, 2000).

Jugé équivoque dès sa création, le titre de "baccalauréat " accolé à l'adjectif "professionnel " apparaît encore plus usurpé lorsque l'échec des titulaires du diplôme dans l'enseignement supérieur devient visible. Il incite du reste les enseignants du supérieur à ne pas considérer les étudiants issus d'un bac pro comme les autres. L'inquiétude liée à leur montée en puissance parmi les étudiants a d'ailleurs fait couler beaucoup d'encre?.

Si cet échec ne pose au ministère qu'un problème relatif lorsque le bac pro est considéré comme un diplôme d'insertion, il n'en va plus de même lorsqu'il est question de conduire 50 \% d'une génération à un diplôme du supérieur (loi du 23 avril 2005). Pour réduire cet échec et atteindre le nouvel objectif de la politique éducative, un changement d'orientation devient nécessaire dans la politique institutionnelle.

Tout en restant secondaire, l'opportunité de poursuite d'études offerte par le diplôme se normalise. Le bac pro doit cependant conduire au BTS plutôt que vers d'autres cursus, mais la sélectivité à l'entrée en formation pose problème. Après avoir été contraints

9. L'emballement médiatique provoqué par la croissance de leur nombre, depuis 2012, en est un signe tangible; on peut également lire sur le site educpro.fr, les nombreux commentaires que suscite la prise en compte, dans l'enseignement supérieur, d'un public déclaré " nouveau ", alors que le diplôme a déjà trente ans. 
d'accueillir de manière privilégiée les bacheliers technologiques, les enseignants de BTS sont peu favorables à l'accueil de bacheliers professionnels. En 2005, des mentions au bac pro sont créées, contre l'avis des partenaires sociaux, afin que des places soient attribuées d'office en STS aux candidats titulaires des meilleures mentions. À l'époque, c'est dans les BTS où les candidats se font rares que les bacheliers professionnels trouvent le plus facilement une place - principalement certains BTS industriels, pour permettre aux sections de formation de perdurer. L'enjeu pour les équipes enseignantes est donc moins d'accueillir des bacheliers professionnels que de conserver la formation qu'ils dispensent. Il se trouve cependant que ces BTS ne sont pas non plus les plus répandus, car ce sont les BTS tertiaires qui sont les plus diffusés et aussi les plus demandés. Grâce aux opportunités de sélection qu'offre cette affluence, l'obligation d'accueillir des titulaires d'une mention bien ou très bien ne s'avère pas vraiment respectée.

Cette initiative n'ayant pas eu les effets escomptés, la double finalité du bac pro est officiellement proclamée lors de la rénovation de la voie professionnelle. Toutefois, comme cela ne suffit pas non plus, de nouvelles idées voient régulièrement le jour, qu'il s'agisse de la création d'un nouveau diplôme du supérieur réservé aux bacheliers professionnels ou de l'imposition de quotas dans les STS. Ces tentatives montrent à quel point les résistances à l'accueil de bacheliers professionnels dans l'enseignement supérieur sont grandes, la restructuration du bac pro n'ayant pas encore totalement changé son image publique. Il faut préciser qu'elle n’a pas non plus modifié son référentiel, ce qui manifeste une fois de plus l'ambivalence de la politique éducative à l'égard de ce diplôme. Doté d'un nouveau cursus de formation, d'une double finalité et de nouveaux liens avec le BTS, le bac pro n'a pas en effet été totalement renouvelé.

Si par de telles mesures, le ministère répond aux accusations de faux baccalauréat adressées au diplôme, tout en se donnant les moyens de limiter le chômage juvénile et d'élever toujours plus haut le niveau d'éducation et de diplôme, il n'a pas pour autant ôté au diplôme toutes ses ambiguïtés. Peut-être même en a-t-il ajouté. La question se pose ainsi de sa déprofessionnalisation. En fondant leur article sur les résultats d'une enquête particulièrement originale, qui porte sur un angle mort de la recherche sur la voie professionnelle, l'évaluation, c'est justement le caractère professionnel du diplôme que Josiane Paddeu et Patrick Veneau mettent en cause dans ce numéro. L'interrogation est pertinente car à force de vouloir se rapprocher de la filière technologique, le bac pro perd de ses propriétés. Même si elle repose sur un bac pro qui est depuis son origine plus technologique que les autres et ne peut être considéré comme représentatif de l'ensemble des spécialités, l'analyse invite à poser des questions importantes sur la hiérarchie, la catégorisation et la légitimité des différents savoirs, ainsi que sur les liens entre connaissance et action. Questions auxquelles la voie professionnelle est depuis toujours confrontée, mais qui vont bien au-delà de ses diplômes. 


\section{Conclusion}

La manière dont les pouvoirs publics et les chercheurs appréhendent le bac pro révèle qu'il n'est pas encore assimilé à un baccalauréat comme les autres.

En revanche, il possède désormais l'une des principales caractéristiques des diplômes professionnels de l'Éducation nationale : celle d'être soumis à un mouvement permanent de réforme. Il paraît ainsi difficile de ne pas envisager une rénovation de son curriculum dans les prochaines années. Dans cette mesure, une forme de banalisation du diplôme a eu lieu.

Pour sa mise en équivalence avec les deux autres baccalauréats, rien n’est acquis. Il est en outre probable que son rapprochement forcé le subordonne davantage à des logiques scolaires sans lui faire gagner en prestige.

Par ailleurs, le brouillage que produit la politique éducative à force de changement (sans rien modifier cependant à la norme scolaire ni à la hiérarchie des filières d'enseignement et des savoirs) pourrait bien avoir des effets sur le marché du travail, le signal envoyé par le diplôme risquant d'apparaître plutôt confus.

\section{Bibliographie}

Agulhon C. (1994), L'enseignement professionnel. Quel avenir pour les jeunes ?, Paris, Éditions de l'Atelier.

Agulhon C. (1995), "Dessinateur industriel : de l'emploi à la formation ", Formation Emploi, n 52, décembre, pp. 11-28.

Agulhon C. (1997), " Les relations formation-emploi, une quête sans fin : les formations à la plasturgie ", Sociologie du travail, nº 3, pp. 321-345.

Baudelot C. \& Establet R. (1971), L'école capitaliste en France, Paris, Maspéro.

Beaud S. (1996), "Les bacs pro. La désouvriarisation du lycée professionnel ", Actes de la recherche en sciences sociales, $\mathrm{n}^{\circ} 114$, pp. 21-29.

Beaud S. (2002), "Le rêve de retrouver la voie normale : les bacs pro à l'université ", in Moreau G., (coord.), Les patrons, l'Etat et la formation des jeunes, Paris, La Dispute, pp. 215-227.

Beaud S. \& Pialoux M. (2001), "Les bacs pro à l'université. Récit d'une impasse ", Revue française de pédagogie, $\mathrm{n}^{\circ} 136, \mathrm{pp} .87-95$.

Bernard P.-Y. \& Troger V. (2012), " La réforme du bac pro en trois ans ou l'appropriation d'une politique éducative par les familles populaires ", Éducation et Sociétés, $\mathrm{n}^{\circ} 30$, pp. 131-143. 
Blöss T. \& Ehrlich V. (2000), "Les nouveaux acteurs de la sélection universitaire : les bacheliers technologiques en question ", Revue française de sociologie, $\mathrm{n}^{\circ}$ 41-4, pp. 747-775.

Bourdieu P. \& Passeron J.-C. (1970), La reproduction, Paris, Éditions de Minuit.

Bouyx B. (1996), "Les diplômes de l'enseignement technologique et professionnel : 1880-1995 ", Éducation et Formations, n 45, pp. 71-78.

Brucy G. (1998), Histoire des diplômes de l'enseignement technique, Paris, Belin.

Cadet J.-P. et alii (2014), Les emplois de la vente et du commerce : quelles évolutions et quelles perspectives pour les deux baccalauréats professionnels?, CPC-Études $n^{\circ} 1$, ministère de l'Éducation nationale.

Campinos-Dubernet M. (1995), "Baccalauréat professionnel, une innovation ? ", Formation Emploi, $\mathrm{n}^{\circ}$ 49, pp. 3-29.

Charlot B. (1999), Le rapport au savoir en milieu populaire. Une recherche dans les lycées professionnels de banlieue, Paris, Anthropos.

Chauvel L. (1998), Le destin des générations. Structure sociale et cohortes en France au XXème siècle, Paris, PUF.

Cizeau T. (2013), "Le CAP, un échauffement pour aller plus loin ?, in Brucy G., Maillard F. \& Moreau G. (dir.), Le CAP, un diplôme du peuple, Rennes, PUR pp. 267-279.

Dagot C. \& Dassié V. (2014), « L'injonction au projet chez les jeunes en baccalauréat professionnel : entre soumission et prise en main fragile de son destiné, Formation Emploi, $\mathrm{n}^{\circ} 128$, pp. 7- 29.

Depoilly S. (2014), Filles et garçons au lycée pro. Rapport à l'école et rapport de genre, Rennes, PUR.

Eckert H. \& Hillau B. (1993), «De la diversification du niveau IV de formation. Les paradoxes de la seconde chance ", L'orientation scolaire et professionnelle, 22, $\mathrm{n}^{\circ} 2$, pp. $147-167$.

Eckert H. (1999), "L'émergence d'un ouvrier bachelier. Les "bac pro“ entre déclassement et recomposition de la catégorie des ouvriers qualifiés ", Revue française de sociologie, vol. 40-2, pp. 227-253.

Figeat M. (1996), Le bac pro : un espoir ou un leurre?, INRP.

Formation Emploi (1995), Dossier «Baccalauréat professionnel », n 49, janvier-mars, La Documentation française.

Fourcade B. (1997), « La création des premiers baccalauréats professionnels de la métallurgie : maintenance, productique, électrotechnique ", in Möbus M. \& Verdier E., 
Les diplômes professionnels en Allemagne et en France. Conception et jeux d'acteurs, Paris, L'Harmattan, pp. 105-128.

Grignon, C. (1971). Lordre des choses. Les fonctions sociales de l'enseignement technique. Éditions de Minuit, Paris

Jellab A. (2001), Scolarité et rapport aux savoirs en lycée professionnel, Paris, PUF.

Jellab A. (2008), Sociologie du lycée professionnel. L'expérience des élèves et des enseignants dans une institution en mutation, Toulouse, Presses du Mirail.

Maillard F. (2005), "L'ambivalence de la politique éducative : le CAP entre déclin et relance ", Formation Emploi, n' ${ }^{\circ}$ 9, pp. 65-78.

Maillard F. (2007), "Vingt ans de politique des diplômes. Un mouvement constant de réforme ", Éducation et Formations, n 75 , pp. 27-36.

Maillard F. (2010,) Enquête sur les premiers pas du bac pro en 3 ans dans le champ tertiaire, CPC-Études $\mathrm{n}^{\circ} 3$, ministère de l'Éducation nationale.

Maillard F. (2011), "La fin du BEP ? ", in Millet M. \& Moreau G., La société des diplômes, Paris, La Dispute, pp. 131-145.

Maillard F. (2013), « Les diplômes professionnels de l'Éducation nationale entre scolarisation et professionnalisation ", Les Dossiers des Sciences de l'Éducation, n 30, pp. 35-52.

Ministère de l'Éducation nationale, (1999, 2007, 2014), Repères et références statistiques.

Moreau G. (1995), "La mixité dans l'enseignement professionnel », Revue française de pédagogie, $\mathrm{n}^{\circ} 110$, pp. 17-25.

Moreau G. (2003), Le monde apprenti, Paris, La Dispute.

Mouy P. \& Veneau P. (1995), "Des objectifs à la réalité. Les bacheliers professionnels industriels ", Formation Emploi, n 49, pp. 91-103.

Palheta U. (2011), " Le collège divise. Appartenance de classe, trajectoires scolaires et enseignement professionnel », Sociologies, Vol. 21, pp. 363-386.

Palheta U. (2012), La domination scolaire. Sociologie de l'enseignement professionnel et de son public, Paris, PUF.

Pillet M. (1995), «La création du baccalauréat professionnel. Les processus de décision », Formation Emploi, $\mathrm{n}^{\circ}$ 52, pp. 87-99.

Prost A. (2011), "La création du baccalauréat professionnel. Histoire d'une décision », in Millet M. \& Moreau G., La société des diplômes, Paris, La Dispute, pp. 113-129.

Quenson E. (2009), "Les diplômes transversaux peinent à s’imposer sur le marché du travail », Formation Emploi, n 106, pp. 25-39. 
Solaux G. (1995), « Le baccalauréat professionnel et son curriculum », Formation Emploi, $\mathrm{n}^{\circ} 49$, pp. 31-45.

Tanguy L. (2013), « Mort et résurrection du CAP », in Brucy G., Maillard F. \& Moreau G. (dir.), Le CAP, un diplôme du peuple, Rennes, PUR, pp. 43-57.

Tanguy L. (1991), Quelle formation pour les ouvriers et les employés en France?, Rapport pour le Secrétariat d'État à l'enseignement technique, La Documentation française.

Veneau P. (1997), "Construction des référentiels et analyse prospective du travail : l'exemple de l'usinage mécanique ", in Möbus M. \& Verdier E., Les diplômes professionnels en Allemagne et en France. Conception et jeux d'acteurs, Paris, L'Harmattan, pp. 163-173.

Zarifian P. (1993), "Le baccalauréat professionnel : de quelques problèmes non résolus ", L'orientation scolaire et professionnelle, 22, $\mathrm{n}^{\circ} 2$, pp. 179-188. 